\title{
Discussion and Inspiration of US military information and communication documents
}

\author{
Chun yong Shang ${ }^{1}$ \\ ${ }^{1}$ No.45 Jiefang Park Road, Jiangan District, Wuhan City, Hubei Province
}

\begin{abstract}
Under the condition of informatization, the writing of information and communication documents should be fast and timely, accurate, concise, safe and confidential. The U.S. military's information and communication documents are no exception in drafting. Due to the differences in the establishment system, command system and operational concept, the U.S. military's information and communication documents have their own unique features. The U.S. military's information and communication documents pay attention to refining, accuracy, different levels of use, standards and specifications. It is very useful to study the US military's information and communication documents for the preparation of our army's document.
\end{abstract}

\section{Classification of US military information and communication documents}

The U.S. military's information and communication documents are divided into peacetime information and communication documents and wartime communication documents according to the time of use, and can be divided into "ordinary", "urgent", "urgent" and "extra urgent" according to the secret level. The codes are RR, $\mathrm{PP}, \mathrm{OO}$ and $\mathrm{ZZ}$, and the time standard is no more than 6 hours, 3 hours, 30 minutes and 10 minutes respectively. According to their uses, they can be divided into eight categories: military briefing, information document, discussion or position document, collaborative document, decision-making document, staff research report, letter, message and so on. This paper focuses on the analysis and introduction of four kinds of information and communication documents, such as military briefing, information document, discussion or position document and staff research report[1].

\subsection{Military briefing}

Military affairs, also known as brief military reports, can be divided into four categories according to the nature of operations: information briefing, staff briefing, decision briefing, and mission briefing[2]. The purpose of the report is to express the facts once and for all by relying on the reference materials that we are familiar with so that the audience can easily understand and master the contents of the report. Military briefing officers usually give a weekly or even daily briefing on a specific subject in a very limited period of time on a topic familiar to an audience. The content of the discussion is very extensive, which can include the current situation, taking specific actions, main combat tasks and so on. The purpose of information briefing is to present the facts to the audience, so that they can understand the current situation, or provide corresponding information according to the specific requirements of the audience. A decision briefing is different from an information briefing, which requires the audience to make a decision not only on the issue, but also on the action to be taken.

\subsection{Information document}

Information documents are usually used to convey information to the operational commander. Communicate commander's instructions between departments. It is usually divided into factual documents, filing memoranda, memoranda, etc. It is mainly used to convey information to the officers who know the situation, report the latest situation, provide materials for Congress, and provide materials for briefing[3].Or answer the consultation from the superior. The filing memorandum mainly records the meeting minutes, telephone conversation and other information. A memorandum generally refers to an informal note given by a s.

\subsection{Discussion or position paper}

The discussion or position paper provides a general guidance for users to discuss and use in consultation, meeting and visiting the headquarters. It mainly includes joint staff documents, key documents, position documents, discussion documents, background documents, etc. The joint staff document is usually divided into position document, talk document and information document. The position document is mainly to summarize the problems, usually using the narrative method, and the degree of detail is mainly based on the

*Corresponding author's e-mail: mast038@bestmaster.xyz 
readers' understanding of the situation. Talking about documents is mainly for the discussion of readers who are very familiar with the meeting. Key documents are often used in unexpected situations at headquarters for discussion. The discussion paper is generally formulated for the future issues to be discussed. [4].It draws conclusions through discussion, promotes the opinions of the headquarters, or achieves other purposes. Background documents mainly provide background materials, current materials and tasks to be completed for specific problems or topics.

\subsection{Staff Research Report}

The staff research report mainly deals with administrative and management issues. The Research Report of the staff officers should specifically list the problems, conclusions and suggestions. The staff research report is mainly used in the Staff Department. It is usually divided into five aspects: problems, assumptions, discussions, conclusions and suggestions.
When describing the problem, we should be concise and accurate, consider from many aspects, think about what kind of difficulties will be encountered in the process of solving problems, and how to solve them. When making false assumptions, we must analyze the problems carefully and comprehensively, and then make reasonable conjectures according to the problems to be solved. We should ensure that the assumptions are based on and can not be fabricated out of thin air [5].In the facts related to the problem, highlight the key issues, and then list the problems in a certain logical order. Pay attention to the details of the change, if you need to make a detailed description of the details, you can put them in the appendix. In the discussion part, we should focus on the analysis of the problem-solving problem, and put forward a number of solutions, including the advantages and disadvantages of the problem. In the discussion, the standard of solving the problem should be given, and the feasibility of each scheme should be checked. In the conclusion part, the best scheme should be given. As shown in in table 1.

Table 1 .staff research report process

\begin{tabular}{ll}
\hline Staff Research Report & Action required for staff study report \\
\hline problem & Understanding problems \\
\hline $\begin{array}{l}\text { Hypothesis } \\
\text { Facts related to the problem }\end{array}$ & Collect necessary information \\
\hline discuss & $\begin{array}{l}\text { Study possible solutions } \\
\text { Analysis of possible solutions }\end{array}$ \\
\hline $\begin{array}{l}\text { Conclusion } \\
\text { proposal }\end{array}$ & Choose the best solution \\
\hline
\end{tabular}

\section{Writing characteristics of US military information and communication documents}

\subsection{The standardization of document writing}

The standardization of US military information and communication documents is mainly reflected in: according to the characteristics of the documents, the contents of the documents are clarified. For example, the main points in the discussion and position documents are mainly divided into five aspects: outline, background, discussion, key points, and staff opinions. The position instrument is divided into four parts: outline, purpose, discussion and suggestion. In terms of stylistic rules, the U.S. military divides information and communication documents into fixed format part and variable format part. The fixed format part has been prepared in advance and can be filled in as required in the given document content form. We should try to be objective, impartial and unbiased, and avoid using modified sentences. When answering questions, we should keep objective and answer questions without emotion. When drafting a message, the U.S. military strives to be accurate in the content of the message. If the word "no" appears in the message, the word "repeat, no" should be added. Make sure that there is no action to the contrary even if "no" is omitted. In addition, it is also required to list the contents of reference in the message. All the reference contents are listed in the first part of the message, and internal information is not used as far as possible. In the summary of action instrument, it is stipulated that the last content of the summary instrument is annex and recommendation.

\subsection{The conciseness of document expression}

The U.S. military's information and communication documents strive to be concise and clear in expression. The most effective communication of the U.S. military is to use the most common words that can express the meaning, rather than those words that are long and not commonly used. In the first or second sentence of the passage, write suggestions, conclusions or reasons for writing this article. In the use of voice, active voice should be used, short sentences (less than 15 words), small words (less than 3 syllables), paragraphs no more than 1 inch long, spelling, grammar and punctuation should be used accurately. Generally, the first person and the second person are used as the subject of a sentence. The length of each document is also limited. The joint 
staff action document should adopt simple narrative style, direct statement and active sentence, with the length of no more than two pages. The key document should use simple sentence pattern, preferably no more than 2 pages. In order to be concise and clear in background documents, outline should be used instead of complete sentences. Details can be attached to the text as an appendix or a schedule. On the one hand, the purpose is to make the article concise and logical so that readers can understand it easily; on the other hand, it can save the commander's time through careful writing.

\subsection{The level of document writing}

The hierarchy of the use of US military information and communication documents mainly shows that commanders at different levels use different documents. Military briefing is used by operational officers to a specific group of targets. In the use of the requirements of high frequency, basically once a week or even daily. In the use of materials, the main source is usually very familiar with the information. However, information documents are mainly used to transmit information to the chief executive, and between staff departments when no reply is required. Staff action documents are mainly used by staff members. Thirdly, different information communication documents are used in different occasions. Information briefing and decision briefing are mainly used for peacetime, mission briefing is mainly used for combat operations, and also for briefing training tasks under simulated combat conditions. A discussion or position paper used for discussion during consultations, meetings, and visits to headquarters. The coordination document is mainly used for the coordination of daily work within the Staff Department. The decision-making documents are mainly used to submit the advice of the staff for decision-making or formal approval, and the staff research report is mainly used to solve the administrative and management problems. The routine reports of staff officers can be used generally, but the comprehensive reports recorded in memoranda and coordination documents can only be used by the Joint Staff Committee.

\section{Enlightenment of US military information and communication documents on our army's document writing}

Learning from other people's strong points will help to make up for our army's shortcomings. First, our army's documents should strive to be concise. In terms of language expression, we should reduce the content of abstract and general qualitative description, and use operable quantitative description language to appropriately and completely express the determination and intention of communication commanders, so as to improve the accuracy of information and communication documents. Second, it is necessary to give more prominence to the subjective initiative of staff officers. In the process of decision-making and preparation of operational orders, if there is no urgent and necessary situation, it should not be specified too detailed, so as not to affect the initiative of chief officers of staff officers. Staff should pay attention to both principle and flexibility in drafting documents. Through the drafting of documents, the U.S. Army clearly defines the responsibilities of staff officers, which is easy to cultivate their resourcefulness and resourcefulness, which is worth learning and learning. Third, our army's information and communication documents should pay more attention to professionalism in the process of drafting. We should pay attention to technicality, try to reduce the technology of "administrative command content", increase the proportion of "technical content", so as to improve the technical content of information and communication documents. Fourth, the information and communication documents of our army should pay more attention to actual combat in the process of drafting, accurately reflect the characteristics and laws of communication support and information service activities under the information conditions, pay attention to the contents of the documents, and strive to be concise and practical.

\section{References}

1. Liu Weiguo, Ruan Yongjun, et al. (2001) guide for officers of the United States joint staff. PLA press. 62-68.

2. Huang Jianming, Zhao Ge, Zhou Jing, et al. (2011) joint communications information system. Military Science Press. 34-37.

3. Zeng Qinghua, Zhang Jianjun, et al. (2008) joint operations communications staff service. Communication Command College. 308-315.

4. Wu Yin. (2017) characteristics and writing inspiration of military documents of the US military. Beijing Evening News . 